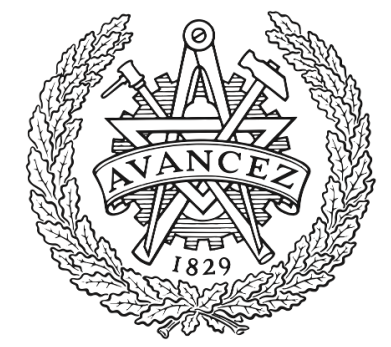

CHALMERS

UNIVERSITY OF TECHNOLOGY

\title{
Tensor Decomposition Based Beamspace ESPRIT for Millimeter Wave MIMO Channel Estimation
}

Downloaded from: https://research.chalmers.se, 2023-04-26 13:44 UTC

Citation for the original published paper (version of record):

Wen, F., Garcia, N., Witrisal, K. et al (2018). Tensor Decomposition Based Beamspace ESPRIT for Millimeter Wave MIMO Channel Estimation. 2018 IEEE Global Communications Conference, GLOBECOM 2018 - Proceedings. http://dx.doi.org/10.1109/GLOCOM.2018.8647176

N.B. When citing this work, cite the original published paper. 


\title{
Tensor Decomposition Based Beamspace ESPRIT for Millimeter Wave MIMO Channel Estimation
}

\author{
Fuxi Wen*, Nil Garcia*, Josef Kulmer ${ }^{\dagger}$, Klaus Witrisal ${ }^{\dagger}$, Henk Wymeersch* \\ ${ }^{*}$ Department of Electrical Engineering, Chalmers University of Technology, SE-412 96, Göteborg, Sweden \\ Email: \{fuxi, nilg, henkw\}@ chalmers.se \\ ${ }^{\dagger}$ Signal Processing and Speech Communication Laboratory, Graz University of Technology, 8010, Graz, Austria \\ Email: \{kulmer,witrisal $\} @$ tugraz.at
}

\begin{abstract}
We propose a search-free beamspace tensor-ESPRIT algorithm for millimeter wave MIMO channel estimation. It is a multidimensional generalization of beamspace-ESPRIT method by exploiting the multiple invariance structure of the measurements. Geometry-based channel model is considered to contain the channel sparsity feature. In our framework, an alternating least squares problem is solved for low rank tensor decomposition and the multidimensional parameters are automatically associated. The performance of the proposed algorithm is evaluated by considering different transformation schemes.
\end{abstract}

Index Terms-Tensor decomposition, beamspace ESPRIT, millimeter wave, MIMO, channel estimation

\section{INTRODUCTION}

Fifth generation (5G) communication networks will likely adopt millimeter wave (mmWave) and massive multiple-inputmultiple-output (MIMO) technologies [1]. In particular, mmwave can provide extremely high data rates to users through dense spatial multiplexing. While mmWave communications also face a number of challenges. Among these, sophisticated beamforming at the transmitter and/or receiver side stands out. To achieve the highly directional links, knowledge of the propagation channel is required. Geometry-based channel modeling is widely used, because it inherently contains the channel sparsity feature. The dominant multipath components can be parametrized in terms of their azimuth and elevation angles at the transmitter and receiver, as well as the corresponding propagation delays and Doppler shifts. Under certain conditions, estimation of these channel parameters gives rise to a multidimensional harmonic retrieval problem. Among the available techniques, maximum-likelihood estimator attains optimum performance in the presence of white Gaussian noise, but with heavy computational load because a multidimensional search is required. Alternatively, subspace method achieves a good balance between accuracy and complexity [2]. State-ofthe-art subspace methods include multiple signal classification (MUSIC) [3], estimation of signal parameters via rotational invariance techniques (ESPRIT) [4], matrix pencil [5], principalsingular-vector utilization for modal analysis [6] and their variants.

In the traditional subspace-based approaches, the $R$-D signals are stored in matrices by stacking operations. However, the multidimensional grid structure inherent in the data is ignored for such a representation. Tensor is a natural approach to store and manipulate multidimensional data $(R \geq 3)$. It can be thought of as a $R$-D array, whereby the order of a tensor is the number of its modes or dimensions; these may include space, time, frequency, trials and classes [7]. Many real-world multi-way data are lying on a low dimensional subspace. Low rank tensor decomposition is a powerful technique to capture the underlying latent structure of the data [8]. CANDECOMP/PARAFAC (CP) and Tucker are two widely used techniques for low rank tensor decomposition. CP decomposes a tensor as a sum of rank-one tensors, and the Tucker decomposition is a higher-order extension of principal component analysis [9]. With the development of tensor decomposition techniques, the subspace methods have been extended to their multi-dimensional variants, such as $R$-D MUSIC [10], multidimensional folding (MDF) [11] and tensor-ESPRIT [12].

Comparing with operation in element space, beamspace model offers a compromise between system performance and hardware complexity [13]. It has a number of advantages, including reduced computational complexity [14], lower signal-to-noise ratio (SNR) resolution thresholds, robustness to sensor perturbations, and deviations from the assumed noise model [15]. Recently, 3-D beamspace ESPRIT is developed for channel estimation of a hybrid mmWave massive MIMO system [16]. The model is described in matrix framework and singular-value decomposition (SVD) is applied to obtain the signal subspace. It is interesting to note that, low-rank tensor decomposition-aided channel estimation for mmWave MIMO-OFDM systems is developed in [17]. However, for each dimension, one dimensional search is required and the complexity of the spectral search may still be unacceptably high for real-time problems [2]).

In this paper, we propose a search-free $R$-D beamspace tensor-ESPRIT algorithm for mmWave channel estimation. The proposed approach is higher-order singular value decomposition (HOSVD) based and it is the $R$-D generalization of the beamspace-ESPRIT method [18]. The standard tensorESPRIT in element space is achieved by using an identity matrix. Furthermore, multidimensional parameter association is critical but challenging for both $5 \mathrm{G}$ communications and localization. For the proposed approach, the $R$-D parameters are automatically associated. A comparison of the relevant subspace algorithms is shown in Table I. 
TABLE I

A COMPARISON OF THE RELATED SUBSPACE ALGORITHMS

\begin{tabular}{l|c|c}
\hline & Element space & Beamspace \\
\hline Matrix framework & {$[3]-[6]$, etc. } & {$[14]-[16],[18],[19]$, etc. } \\
\hline Tensor framework & {$[11],[12]$, etc. } & {$[17]$, proposed } \\
\hline
\end{tabular}

\section{Preliminary and System Model}

We use $(\cdot)^{H},(\cdot)^{*}$ and $(\cdot)^{-1}$ to denote Hermitian transpose, complex conjugate and matrix inverse, respectively. The set of unitary matrices of size $m \times n$ is denoted as $\mathcal{O}_{m \times n}$. In this paper, we follow the tensor operations defined in [20]. The $\left(i_{1}, i_{2}, \cdots, i_{R}\right)$ entry of an $R$-D tensor $\mathcal{A}$ is denoted as $a_{i_{1}, i_{2}, \cdots, i_{R}}$. Scalar product of two tensors is defined as

$$
<\mathcal{A}, \mathcal{B}>=\sum_{i_{1}} \sum_{i_{2}} \cdots \sum_{i_{R}} b_{i_{1}, i_{2}, \cdots, i_{R}}^{*} a_{i_{1}, i_{2}, \cdots, i_{R}} .
$$

The Frobenius norm of a tensor $\mathcal{A}$ is written as

$$
\|\mathcal{A}\|_{F}=\sqrt{\langle\mathcal{A}, \mathcal{A}\rangle} .
$$

The product of a tensor $\mathcal{A} \in \mathbb{C}^{I_{1} \times I_{2} \times \cdots \times I_{R}}$ and a matrix $\mathbf{U} \in \mathbb{C}^{J_{r} \times I_{r}}$ along the $r$ th dimension is denoted by $\mathcal{A} \times{ }_{r} \mathbf{U}$, it is an $\left(I_{1} \times I_{2} \times \cdots \times I_{r-1} \times J_{r} \times I_{r+1} \times \cdots \times I_{R}\right)$-tensor and the entries are defined as

$$
\begin{aligned}
& \left(\mathcal{A} \times_{r} \mathbf{U}\right)_{i_{1}, i_{2}, \cdots, i_{r-1}, j_{r}, i_{r+1}, \cdots, i_{R}} \\
& =\sum_{i_{r}} a_{i_{1}, i_{2}, \cdots, i_{r-1}, i_{r}, i_{r+1}, \cdots, i_{R}} u_{j_{r}, i_{r}} .
\end{aligned}
$$

And $\mathcal{A} \times{ }_{r=1}^{R} \mathbf{U}_{r}$ denotes the product of a tensor $\mathcal{A}$ and matrices $\mathbf{U}_{1}, \mathbf{U}_{2}, \cdots, \mathbf{U}_{R}$ along the $r=1$ to $R$ th dimension [21]. Matrix $[\mathcal{A}]_{(r)} \in \mathbb{R}^{I_{r} \times\left(I_{1} \cdots I_{r-1} I_{r+1} \cdots I_{R}\right)}$ denotes the $r$ th unfolding of $\mathcal{A}$ and $\mathcal{I}_{L}^{R} \in \mathbb{R}^{L \times L \times \cdots \times L}$ is an $R$-D identity tensor whose $(l, l, \cdots, l)$ entry equals one and zero otherwise, $l=1,2, \cdots, L$.

We consider the following system model. The transmitter is equipped with a uniform rectangular array (URA) with $\left(M_{1} \times M_{2}\right)$ elements. The coordinate of the $\left(m_{1}, m_{2}\right)$-th antenna element is $\left(\frac{\lambda}{2} m_{1}, \frac{\lambda}{2} m_{2}, 0\right)$ in three dimensional Cartesian coordinate systems, where $\lambda$ is the wavelength of the carrier frequency. In both directions, the inter-element spacing is $\lambda / 2$. The origin is the array reference point. The receiver is also equipped with a URA with $\left(M_{3} \times M_{4}\right)$ elements, the coordinate of the $\left(m_{3}, m_{4}\right)$-th antenna element is $\left(\frac{\lambda}{2} m_{3}, \frac{\lambda}{2} m_{4}, 0\right)$. The corresponding orthogonal frequency division multiplexing for MIMO channels (MIMO-OFDM) with $M_{5}$ subcarriers can be described as a multipath geometry-model with $L$ paths. Each path $l$ is characterized by its angle of arrival $\left(\theta_{R, l}, \varphi_{R, l}\right)$, angle of departure $\left(\theta_{T, l}, \varphi_{T, l}\right)$, delay $\tau_{l}$ and complex gain $\alpha_{l}$ [13].

Tensor measurements $\mathcal{X}$ is denoted as

$$
\mathcal{X}=\mathcal{I}_{L}^{6} \times{ }_{r=1}^{6} \mathbf{A}_{r}+\mathcal{N} \in \mathbb{C}^{M_{1} \times M_{2} \times \cdots \times M_{6}},
$$

where $\mathcal{N}$ denotes the noise tensor and $M_{6}$ is the number of measurements. For dimension $r=1,2, \cdots, 5$,

$$
\mathbf{A}_{r}=\left[\begin{array}{llll}
\mathbf{a}_{r, 1} & \mathbf{a}_{r, 2} & \cdots & \mathbf{a}_{r, L}
\end{array}\right] \in \mathbb{C}^{M_{r} \times L},
$$

with $\mathbf{a}_{r, l}=\left[\begin{array}{llll}e^{j \omega_{r, l}} & e^{j 2 \omega_{r, l}} & \cdots & e^{j M_{r} \omega_{r, l}}\end{array}\right]^{T}$, and frequencies

$$
\begin{aligned}
& \omega_{1, l}=\pi \cos \left(\theta_{T, l}\right) \sin \left(\varphi_{T, l}\right), \\
& \omega_{2, l}=\pi \sin \left(\theta_{T, l}\right) \sin \left(\varphi_{T, l}\right), \\
& \omega_{3, l}=\pi \cos \left(\theta_{R, l}\right) \sin \left(\varphi_{R, l}\right), \\
& \omega_{4, l}=\pi \sin \left(\theta_{R, l}\right) \sin \left(\varphi_{R, l}\right), \\
& \omega_{5, l}=2 \pi \tau_{l} \Delta_{f},
\end{aligned}
$$

where $\Delta_{f}$ is the neighboring subcarrier spacing, angles $\theta_{T, l}, \varphi_{T, l}, \theta_{R, l}$ and $\varphi_{R, l} \in\left(-\frac{\pi}{2}, \frac{\pi}{2}\right)$. For the 6th dimension,

$$
\mathbf{A}_{6}=\left[\begin{array}{llll}
\mathbf{a}_{6,1} & \mathbf{a}_{6,2} & \cdots & \mathbf{a}_{6, L}
\end{array}\right] \in \mathbb{C}^{M_{6} \times L},
$$

with $\mathbf{a}_{6, l}=\left[\begin{array}{llll}\alpha_{l}(1) & \alpha_{l}(2) & \cdots & \alpha_{l}\left(M_{6}\right)\end{array}\right]^{T}$. Doppler shift can be considered in a similar way by repeating measurements consecutively in time and a 7-D measurement is assembled.

Beamspace model (hybrid structure) offers a compromise between system performance and hardware complexity. Consider a class of separable 6-D precoders or beamformers [19] that transform the $M_{1} \times M_{2} \times \cdots M_{6}$ element space snapshot into an $N_{1} \times N_{2} \times \cdots N_{6}$ beamspace snapshot, according to

$$
\mathcal{Y}=\mathcal{I}_{L}^{6} \times{ }_{r=1}^{6}\left(\mathbf{W}_{r}^{H} \mathbf{A}_{r}\right)+\mathcal{N} \in \mathbb{C}^{N_{1} \times N_{2} \times \cdots \times N_{6}},
$$

where $\mathbf{W}_{r} \in \mathbb{C}^{M_{r} \times N_{r}}$ is the $r$ th dimension linear transformation matrix with orthogonal columns. The beamspace array manifold is defined as

$$
\mathbf{B}_{r}=\mathbf{W}_{r}^{H} \mathbf{A}_{r} \in \mathbb{C}^{N_{r} \times L} .
$$

Note that measurements obtained for $\mathbf{W}_{r}=\mathbf{I}_{M_{r}}$, correspond to standard element space measurements. $\mathbf{W}_{1}$ and $\mathbf{W}_{2}$ correspond to the precoders used by the transmitter. And $\mathbf{W}_{3}$ and $\mathbf{W}_{4}$ correspond to the beamformer used by the receiver. While for the 5th and 6th dimension, transformation is not applied, $\mathbf{W}_{5}=\mathbf{I}_{M_{5}}$ and $\mathbf{W}_{6}=\mathbf{I}_{M_{6}}$. Our objective is estimating $\omega_{r, l}$, for $r=1, \cdots, 5$ and $l=1, \cdots, L$, from measurements $\mathcal{Y}$ using the proposed beamspace tensor-ESPRIT method.

\section{A. Higher-Order Singular Value Decomposition}

Higher-order orthogonality iteration (HOOI) algorithm [22] can be used to estimate the signal subspace from noisy measurements. It solves the following Frobenius norm minimization problem,

$$
\begin{array}{cl}
\min _{\mathcal{C}, \mathbf{U}_{1}, \cdots, \mathbf{U}_{R}} & \left\|\mathcal{C} \times_{r=1}^{R} \mathbf{U}_{r}-\mathcal{Y}\right\|_{F}^{2} \\
\text { s.t. } & \mathbf{U}_{r} \in \mathcal{O}_{N_{r} \times L}, r=1,2, \cdots, R,
\end{array}
$$

where $\mathcal{C} \in \mathbb{C}^{L \times L \times \cdots \times L}$ is the core tensor and $\mathbf{U}_{r}$ has orthonormal columns. Note that with $\mathbf{U}_{r}, r=1,2, \cdots, R$, fixed, the optimal $\mathcal{C}$ is given by

$$
\mathcal{C}=\mathcal{Y} \times_{r=1}^{R} \mathbf{U}_{r}^{H},
$$

we can eliminate $\mathcal{C}$ by plugging (15) to (14), and obtain the following equivalent problem,

$$
\begin{array}{cl}
\min _{\mathbf{U}_{1}, \cdots, \mathbf{U}_{R}} & \left\|\mathcal{Y} \times_{r=1}^{R}\left(\mathbf{U}_{r} \mathbf{U}_{r}^{H}\right)-\mathcal{Y}\right\|_{F}^{2} \\
\text { s.t. } & \mathbf{U}_{r} \in \mathcal{O}_{N_{r} \times L}, r=1,2, \cdots, R .
\end{array}
$$


HOOI [22] alternatively update $\mathbf{U}_{1}, \cdots, \mathbf{U}_{R}$ by minimizing (16) with respect to one of them while fixing the remaining variables. Let superscript $(\cdot)^{(k)}$ be the estimate at the $k$ th iteration, $\mathbf{U}_{r}^{(k)}$ can be written as

$$
\mathbf{U}_{r}^{(k)}=\arg \max _{\mathbf{U}_{r}}\left\|\mathbf{U}_{r}^{H} \mathbf{Y}_{r}^{(k)}\right\|_{F}^{2} \text {, s.t. } \mathbf{U}_{r} \in \mathcal{O}_{N_{r} \times L},
$$

where

$$
\mathbf{Y}_{r}^{(k)}=\left(\mathcal{Y} \times{ }_{i=1}^{r-1}\left(\mathbf{U}_{i}^{(k)}\right)^{H} \times_{i=r+1}^{R}\left(\mathbf{U}_{i}^{(k-1)}\right)^{H}\right)_{[r]} .
$$

The solution of (17) can be determined using singular-value decomposition, simply set $\mathbf{U}_{r}^{(k)}$ be the matrix containing the left $L$ leading singular vectors of $\mathbf{Y}_{r}^{(k)}$.

\section{B. Tensor-ESPRIT}

We first review the element space tensor-ESPRIT method [12]. The main idea is exploiting the multidimensional shift invariance property of the measurements. For each dimension, the array is divided into two subarrays with same number of elements. The subarrays may overlap and an element may be shared by the two subarrays. Let $\overline{\mathbf{U}}_{r} \in \mathbb{C}^{M_{r} \times L}$ be the subspace spanned by $\mathbf{A}_{r} \in \mathbb{C}^{M_{r} \times L}$, which can be obtained by applying tensor decomposition on $\mathcal{X}$. For the $r$ th dimension, we have

$$
\mathbf{A}_{r}=\overline{\mathbf{U}}_{r} \overline{\mathbf{D}}_{r},
$$

where $\overline{\mathbf{D}}_{r} \in \mathbb{C}^{L \times L}$ is a non-singular matrix. We further define two sub-matrices,

$$
\overline{\mathbf{U}}_{r_{1}}=\overline{\mathbf{J}}_{r_{1}, n} \overline{\mathbf{U}}_{r} \text { and } \overline{\mathbf{U}}_{r_{2}}=\overline{\mathbf{J}}_{r_{2}, n} \overline{\mathbf{U}}_{r},
$$

where $\overline{\mathbf{J}}_{r_{1}, n}$ and $\overline{\mathbf{J}}_{r_{2}, n}$ are two selection matrices,

$$
\begin{aligned}
& \overline{\mathbf{J}}_{r_{1}, n}=\left[\begin{array}{ll}
\mathbf{I}_{M_{r}-n} & \mathbf{0}_{\left(M_{r}-n\right) \times n}
\end{array}\right], \\
& \overline{\mathbf{J}}_{r_{2}, n}=\left[\begin{array}{ll}
\mathbf{0}_{\left(M_{r}-n\right) \times n} & \mathbf{I}_{M_{r}-n}
\end{array}\right],
\end{aligned}
$$

where $\mathbf{I}_{n}$ denotes identity matrix of size $n \times n$ and $\mathbf{0}_{m \times n}$ denotes zero matrix of size $m \times n$. For convenience, we focus on $n=1, \overline{\mathbf{J}}_{r_{1}, 1}$ and $\overline{\mathbf{J}}_{r_{2}, 1}$ are simplified as $\overline{\mathbf{J}}_{r_{1}}$ and $\overline{\mathbf{J}}_{r_{2}}$. Then we have

$$
\overline{\mathbf{J}}_{r_{1}} \mathbf{A}_{r}=\overline{\mathbf{J}}_{r_{2}} \mathbf{A}_{r} \mathbf{\Phi}_{r}
$$

where

$$
\boldsymbol{\Phi}_{r}=\operatorname{diag}\left[\begin{array}{llll}
e^{-j \omega_{r, 1}} & e^{-j \omega_{r, 2}} & \cdots & e^{-j \omega_{r, L}}
\end{array}\right] .
$$

Substituting (19) and (20) into (22), we obtain

$$
\overline{\mathbf{U}}_{r_{1}}=\overline{\mathbf{U}}_{r_{2}} \Psi_{r},
$$

where

$$
\boldsymbol{\Psi}_{r}=\overline{\mathbf{D}}_{r} \boldsymbol{\Phi}_{r} \overline{\mathbf{D}}_{r}^{-1} \in \mathbb{C}^{L \times L} .
$$

The equations in (24) is over-determined. The simplest choice to estimate $\boldsymbol{\Psi}_{r}$ is using least squares method and the resulting closed-form solution is given by

$$
\hat{\mathbf{\Psi}}_{r}=\left(\overline{\mathbf{U}}_{r_{2}}\right)^{\dagger} \overline{\mathbf{U}}_{r_{1}},
$$

where $\dagger$ denotes the Moore-Penrose matrix inverse. Let $\lambda_{r, 1}, \lambda_{r, 2}, \cdots, \lambda_{r, L}$ be the eigenvalues of $\hat{\mathbf{\Psi}}_{r}$, the mode $r$ frequencies are estimated by using

$$
\omega_{r, l}=-\angle\left(\lambda_{r, l}\right), l=1,2, \cdots, L,
$$

where $\angle(\cdot)$ denotes the argument of a complex number.

Note that (27) ignores the correct association of the parameters across the dimensions. In ESPRIT-type algorithms [23], the association is usually achieved by joint approximate eigendecomposition [24] or simultaneous Schur decomposition [25].

\section{III. $R$-D BEAMSPACE TENSOR-ESPRIT}

Before introducing the proposed $R$-D beamspace tensorESPRIT method, let's first define two selection matrices,

$$
\begin{aligned}
& \mathbf{J}_{r_{1}}=\left[\begin{array}{ll}
\mathbf{I}_{N_{r}-1} & \mathbf{0}_{\left(N_{r}-1\right) \times 1}
\end{array}\right], \\
& \mathbf{J}_{r_{2}}=\left[\begin{array}{ll}
\mathbf{0}_{\left(N_{r}-1\right) \times 1} & \mathbf{I}_{N_{r}-1}
\end{array}\right] .
\end{aligned}
$$

In beamspace, the transitional invariance structure in the array manifold is altered by the row transformation $\mathbf{W}_{r}^{H}$, and consequently

$$
\mathbf{J}_{r_{1}} \mathbf{B}_{r} \neq \mathbf{J}_{r_{2}} \mathbf{B}_{r} \mathbf{\Phi}_{r} .
$$

In general, it is difficult to restore this property after beamspace transformation [18]. However, if $\mathbf{W}_{r}$ has a similar shift invariance structure as (22), the lost shift invariance structure can be restored. Similar to (22), we want to find a non-singular $N_{r} \times N_{r}$ matrix $\mathbf{F}_{r}$ to satisfy

$$
\mathbf{J}_{r_{1}} \mathbf{W}_{r}=\mathbf{J}_{r_{2}} \mathbf{W}_{r} \mathbf{F}_{r} .
$$

The least squares estimation of $\mathbf{F}_{r}$ is given by

$$
\hat{\mathbf{F}}_{r}=\left(\mathbf{J}_{r_{2}} \mathbf{W}_{r}\right)^{\dagger} \mathbf{J}_{r_{1}} \mathbf{W}_{r} .
$$

Theorem 1: Let $\mathbf{F}_{r}$ be defined as in (30) and

$$
\mathbf{W}_{r}^{H}=\left[\begin{array}{llll}
\mathbf{w}_{1} & \mathbf{w}_{2} & \cdots & \mathbf{w}_{M_{r}}
\end{array}\right] \in \mathbb{C}^{N_{r} \times M_{r}} .
$$

If there exists a $\mathbf{Q}_{r} \in \mathbb{C}^{N_{r} \times N_{r}}$, such that

$$
\left\{\begin{array}{l}
\mathbf{Q}_{r} \mathbf{w}_{M_{r}}=\mathbf{0}_{N_{r} \times 1}, \\
\mathbf{Q}_{r} \mathbf{F}_{r}^{H} \mathbf{w}_{1}=\mathbf{0}_{N_{r} \times 1},
\end{array}\right.
$$

then

$$
\mathbf{Q}_{r} \mathbf{F}_{r}^{H} \mathbf{B}_{r}=\mathbf{Q}_{r} \mathbf{B}_{r} \boldsymbol{\Phi}_{r}^{H} .
$$

Proof: See Appendix A.

It is worth noting that $\mathbf{Q}_{r}$ in (33) can be found by forming a projection matrix corresponding to the orthogonal subspace of $\mathcal{R}\left\{\mathbf{w}_{M_{r}}, \mathbf{F}_{r}^{H} \mathbf{w}_{1}\right\}$. Then

$$
\hat{\mathbf{Q}}_{r}=\mathbf{I}_{N_{r}}-\mathbf{w}_{M_{r}} \mathbf{w}_{M_{r}}^{H}-\left(\mathbf{F}_{r}^{H} \mathbf{w}_{1}\right)\left(\mathbf{F}_{r}^{H} \mathbf{w}_{1}\right)^{H} .
$$

Comparing (22) and (34), the array shift invariance structure in beamspace is restored. Replacing $\mathbf{B}_{r}$ by the estimated signal subspace $\mathbf{U}_{r}$,

$$
\mathbf{B}_{r}=\mathbf{U}_{r} \mathbf{D}_{r},
$$

where $\mathbf{D}_{r} \in \mathbb{C}^{L \times L}$ is a non-singular matrix. The transformed shift invariance equation then becomes

$$
\mathbf{Q}_{r} \mathbf{F}_{r}^{H} \mathbf{U}_{r}=\mathbf{Q}_{r} \mathbf{U}_{r} \boldsymbol{\Gamma}_{r}
$$


where

$$
\boldsymbol{\Gamma}_{r}=\mathbf{D}_{r} \boldsymbol{\Phi}_{r} \mathbf{D}_{r}^{-1} \in \mathbb{C}^{L \times L} .
$$

For simplicity, least squares technique is used to solve (37). The resulting close-form solution is given by

$$
\hat{\boldsymbol{\Gamma}}_{r}=\left(\mathbf{Q}_{r} \mathbf{U}_{r}\right)^{\dagger} \mathbf{Q}_{r} \mathbf{F}_{r}^{H} \mathbf{U}_{r} .
$$

The $l$ th eigenvalue of $\boldsymbol{\Gamma}_{r}$ is given by $e^{j \omega_{r, l}}$. Frequency $\boldsymbol{\omega}_{r}=$ $\left\{\omega_{r, 1}, \omega_{r, 2}, \cdots, \omega_{r, L}\right\}$ can be estimated from the eigenvalues of $\boldsymbol{\Gamma}_{r}$. Note that each column of $\mathbf{U}_{r}$ is characterized by the associated frequency $\omega_{r, l}$.

As shown in (18), the subspaces $\mathbf{U}_{r}, r=1,2, \cdots, 5$ are alternatively updated. The $l$ th column of $\mathbf{U}_{r}$, for all $r$, corresponds to the same source. For the $r$ th dimension, the $L$ frequencies $\left\{\omega_{r, 1}, \omega_{r, 2}, \cdots, \omega_{r, L}\right\}$ can be estimated jointly from (39), but the association between dimensions is lost. To solve this problem, instead of estimating the frequencies directly from (39), we propose the following parallel estimation scheme, which automatically associates the $R$-D frequencies across dimensions.

Let $\mathbf{u}_{r, l}$ be the $l$ th column of $\mathbf{U}_{r}$, then we have

$$
\varphi_{r, l}=\left(\mathbf{Q}_{r} \mathbf{u}_{r, l}\right)^{\dagger} \mathbf{Q}_{r} \mathbf{F}_{r}^{H} \mathbf{u}_{r, l},
$$

the $l$ th frequency of the $r$ th dimension $\omega_{r, l}$ is obtained from the phase angle of $\varphi_{r, l}$. While for each dimension step (40) is implemented in parallel for $l=1,2, \cdots, L$ and $r=1,2, \cdots, 5$. And $\left\{\omega_{1, l}, \omega_{2, l}, \cdots, \omega_{5, l}\right\}$ are corresponding to the same source, then channel parameters $\left\{\theta_{T, l}, \varphi_{T, l}, \theta_{R, l}, \varphi_{R, l}, \tau_{l}\right\}$ can be recovered from the estimated frequencies, according to (6)-(10). Thus, the multidimensional parameter association problem is solved, and it can also be applied to the standard tensor-ESPRIT.

The proposed beamspace tensor-ESPRIT is summarized in Algorithm 1.

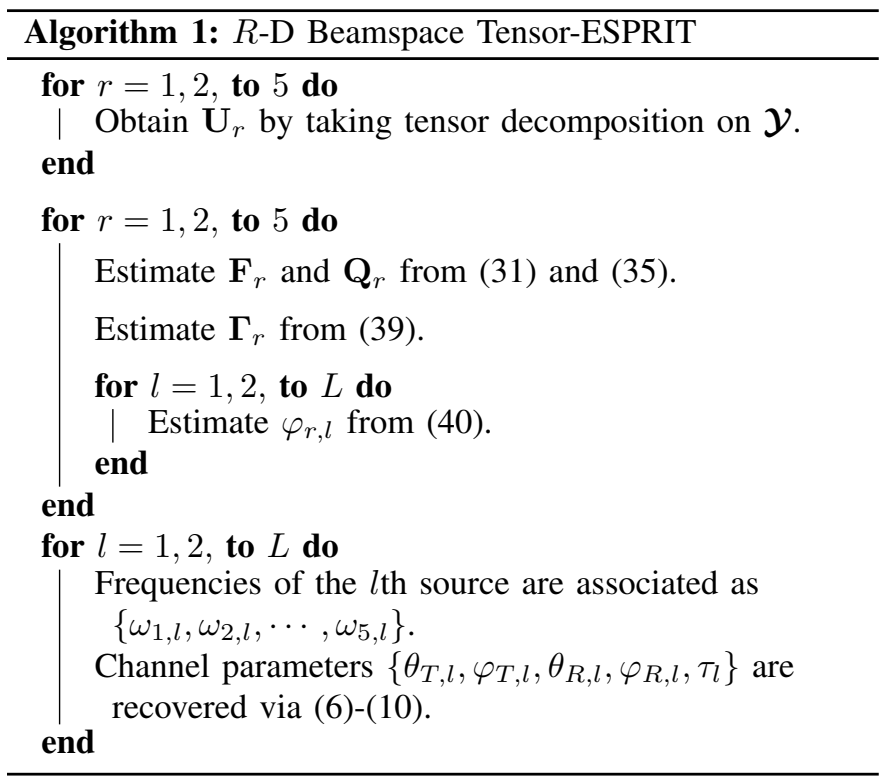

\section{Simulation Setup And Numerical Results}

Numerical simulations have been carried out to evaluate the performance of the proposed method for $R$-D HR in the presence of white Gaussian noise. The performance is assessed by the total root-mean square error (RMSE) on the estimated parameters. The total RMSE of the angle-of-departure and the angle-of-arrival is defined as

$$
\operatorname{RMSE}=\sqrt{\frac{1}{4 L} \mathbb{E}_{t}\left\{\sum_{r=1}^{4} \sum_{l=1}^{L}\left(\omega_{r, l}-\hat{\omega}_{r, l}\right)^{2}\right\}}
$$

where $\hat{\omega}_{r, l}$ is an estimate of $\omega_{r, l}$, and $\mathbb{E}_{t}$ denotes the average based on $t=500$ Monte-Carlo trials. The RMSE performance of the delays can be calculated in a similar way.

In the following test, 5-D channel estimation from noisy observations is considered. Both transmitter and receiver consist of a URA with $8 \times 8$ elements, $M_{1}=M_{2}=M_{3}=M_{4}$, the number of subcarriers is $M_{5}=8$ and the number of measurements is $K=1$. The subcarrier spacing is $\Delta_{f}=2$ $\mathrm{MHz}$ and the signal-to-noise ratio (SNR) is defined as

$$
\mathrm{SNR}=\frac{\|\mathcal{Y}-\mathcal{N}\|_{F}^{2}}{\|\mathcal{N}\|_{F}^{2}} .
$$

For dimensions $r=1,2,3,4$, the $n_{r}$-th column of transformation matrix $\mathbf{W}_{r} \in \mathbb{C}^{M_{r} \times N_{r}}$, is constructed as

$$
\mathbf{w}_{n_{r}}=\left[\begin{array}{llll}
1 & e^{j \pi \omega_{n_{r}}} & \cdots & e^{j \pi\left(M_{r}-1\right) \omega_{n_{r}}}
\end{array}\right]^{T} \in \mathbb{C}^{N_{r} \times 1},
$$

where random number $\omega_{m_{r}}$ is drawn from uniform distribution $\mathcal{U}(0,1)$. For the 5 th dimension, $\mathbf{W}_{5}=\mathbf{I}_{M_{5}}$.

\section{A. Channel Estimation and Parameter Association}

In the first two tests, the three sets of channel parameters $\left(\theta_{T, l}, \varphi_{T, l}, \theta_{R, l}, \varphi_{R, l}, \tau_{l}\right)$ are

$$
\begin{array}{ll}
l=1: & \left(-14^{\circ}, 8^{\circ},-20^{\circ},-30^{\circ}, 30 \mathrm{~ns}\right), \\
l=2: & \left(60^{\circ}, 65^{\circ}, 10^{\circ}, 50^{\circ}, 40 \mathrm{~ns}\right), \\
l=3: & \left(-70^{\circ}, 75^{\circ}, 15^{\circ},-5^{\circ}, 50 \mathrm{~ns}\right) .
\end{array}
$$

For beamspace T-ESPRIT, the data dimension is $N_{1} \times N_{2} \times$ $N_{3} \times N_{4} \times N_{5}$, and different values of $N_{r}$ are considered for all $r$. Fig. 1 plots the RMSE under different SNRs. The proposed beamspace method is compared with element space T-ESPRIT algorithm. We observe that RMSE is reduced by increasing the size of the transformation matrix, and it is close to the RMSE of element space ESPRIT when a larger $N_{r}$ is selected.

A correct parameter association is critical but challenging in $R$-D channel estimation. In this second test, the automatic association example of the proposed method is shown. Beam dimension $N_{1} \times N_{2} \times N_{3} \times N_{4} \times N_{5}$ is $8 \times 8 \times 8 \times 8 \times 8$, and SNR is $20 \mathrm{~dB}$. As shown in Fig. 2, the 5-D parameters are associated for the proposed beamspace tensor-ESPRIT method. But with some outliers, it is caused by the way we generate the transformation matrix $\mathbf{W}_{r}$. As shown in (43), if two $\omega_{m_{r}}$ are close to each other, then the information provided by them is redundant. It may cause rank deficiency of the transformation matrix. 


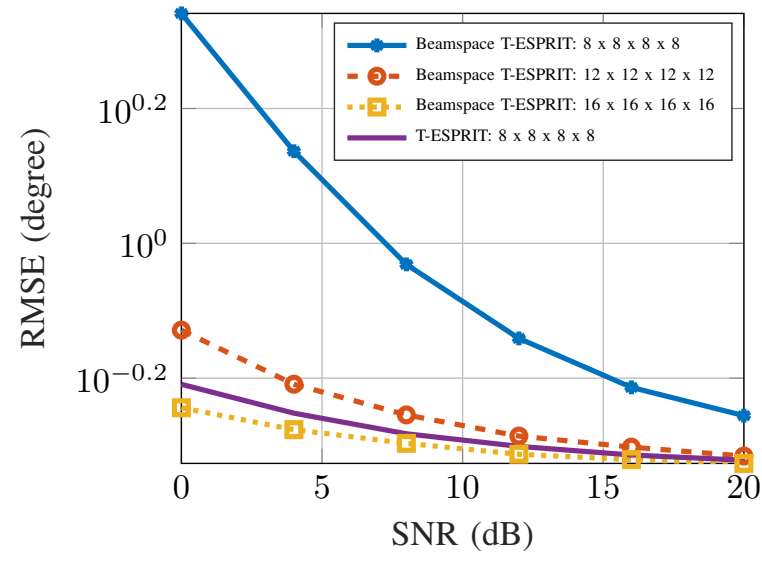

(a) Angle

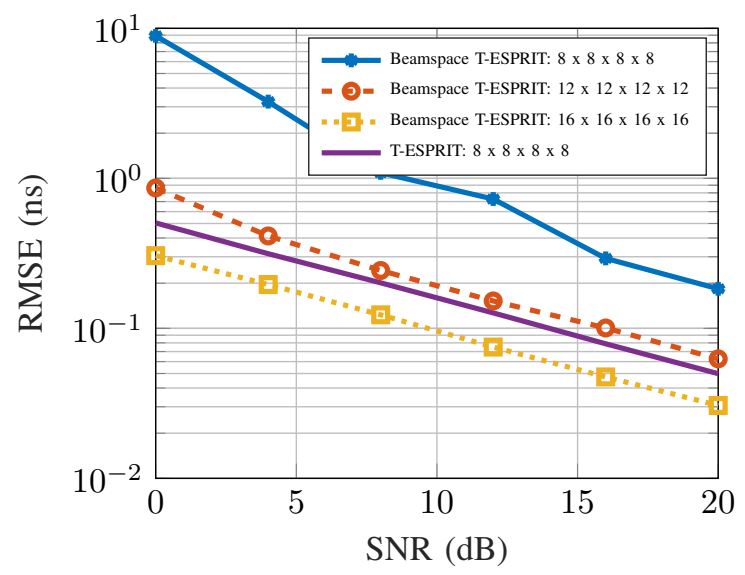

(b) Delay

Fig. 1. 5-D channel estimation performance versus SNR for the proposed beamspace tensor-ESPRIT method.

\section{B. Numerical Results for Distributed Sources}

In the following simulations, two widely used distributed source models, Gaussian distributed [26] and Lambertian scattering [27] are considered.

1) Gaussian Distributed Model: In this simulation, we assume three Gaussian incoherently distributed sources. For each path, 20 scatters are generated, the 5-D channel parameters of the scatters are drawn independently from a Gaussian distribution. Central angle and delay are same as the simulation setup in Section IV-A. Different standard deviation of angular and delay spreads are considered. In our evaluation we consider various levels of angular and delay spread. As shown in Fig. 3, deterioration of RMSE performance occurs for T-ESPRIT and the proposed method with increased angular and delay spread levels. Again RMSE is reduced by increasing the size of the transformation matrix.

2) Lambertian Scattering Model: In this experiment we evaluate the performance of estimating parameters of specular reflections. Specular reflections originate at flat surfaces and are characterized by equal incident and reflected angle at the surface. To ensure a realistic scenario we deteriorate

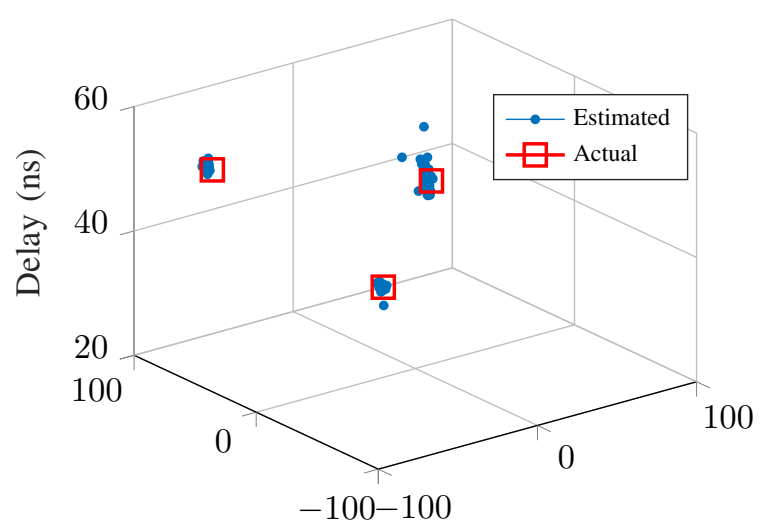

Tx: elevation (degree)

Tx: azimuth (degree)

(a) The channel parameters are $\theta_{T, l}, \phi_{T, l}$ and $\tau_{l}$ and $l=1,2,3$.

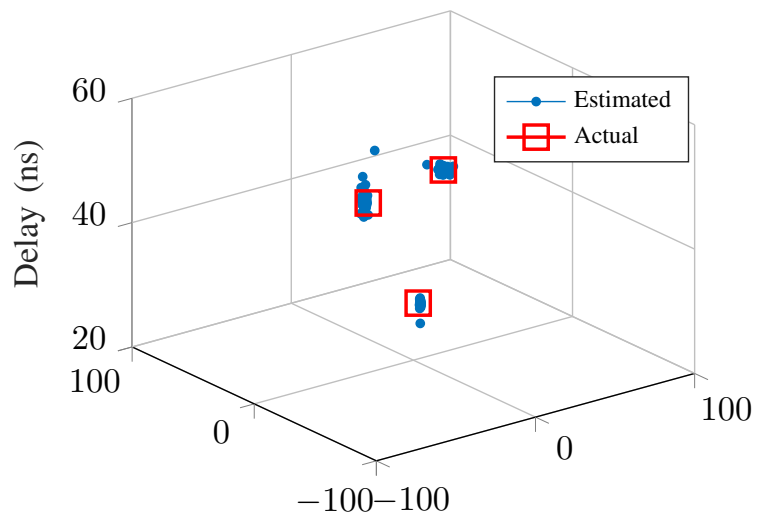

Rx: elevation (degree) Rx: azimuth (degree)

(b) The channel parameters are $\theta_{R, l}, \phi_{R, l}$ and $\tau_{l}$ and $l=1,2,3$.

Fig. 2. Parameter association (5-D) example for the proposed beamspace tensor-ESPRIT method.

the specular reflection with diffuse scattering stemming from rough surfaces. We describe the impact from diffuse scattering by placing scatter points at each reflective surface. The scatter points are drawn from a scattering distribution which is calculated based on Lambertian scattering.

In our simulations we consider a street scenario with two buildings facing each other with a distance of $20 \mathrm{~m}$ (see Figure 4). The transmitter is located next to building 1 with a distance of $2 \mathrm{~m}$ at height $5 \mathrm{~m}$; and the receiver is located next to building 2 with distance $6 \mathrm{~m}$ and height $2 \mathrm{~m}$. Both transceivers are separated by $12 \mathrm{~m}$ and $3 \mathrm{~m}$ along the $y$ - and $x$-axis. They are equipped with an URA of $8 \times 8$ elements, with $M_{5}=8$, $K=1, \Delta_{f}=2 \mathrm{MHz}$. Assuming a single, specular reflection at the ground surface and each building, we get a line-of-sight (LOS) path $(l=1)$, a ground reflection $(l=2)$ and reflections at both buildings $(l=3,4)$. The parameters of the LOS and 


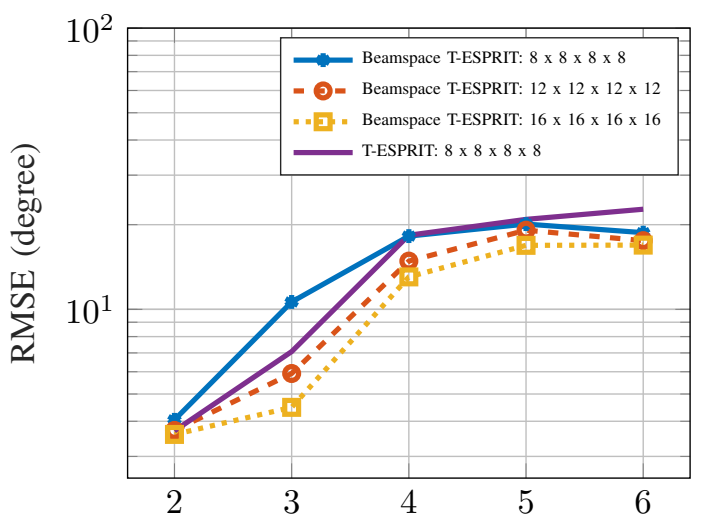

Standard deviation of angular spread (degree)

(a) RMSE versus angular spread

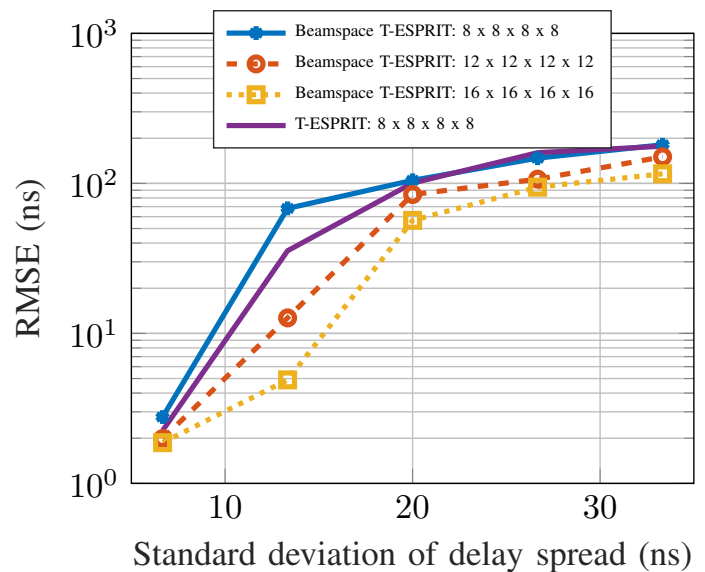

(b) RMSE versus delay spread

Fig. 3. 5-D channel estimation performance versus angular and delay for the proposed beamspace tensor-ESPRIT method under a Gaussian distributed model.

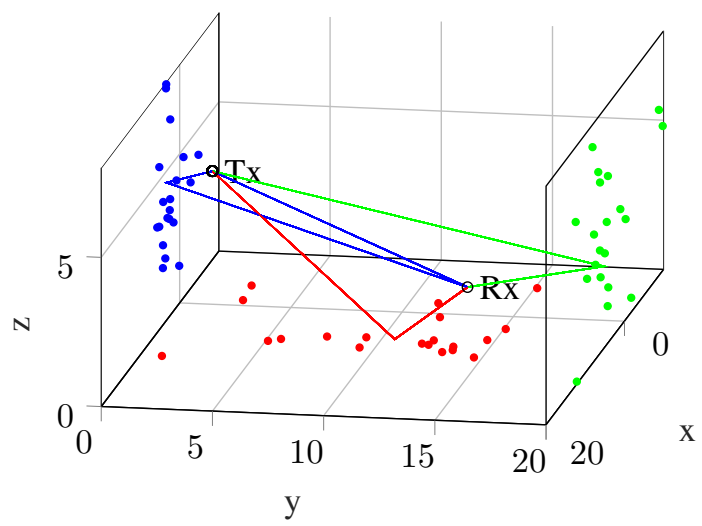

Fig. 4. Lambertian scattering. Illustration of street scenario consisting of a line of sight plus 3 reflections, in total $L=4$ paths. Dots represent scatter points.

the specular reflections are

$$
\begin{array}{ll}
l=1: & \left(78.69^{\circ}, 101.10^{\circ},-101.31^{\circ}, 78.90^{\circ}, 51.9 \mathrm{~ns}\right), \\
l=2: & \left(78.69^{\circ}, 114.59^{\circ},-101.31^{\circ}, 114.59^{\circ}, 56.0 \mathrm{~ns}\right), \\
l=3: & \left(-80.03^{\circ}, 98.86^{\circ},-98.97^{\circ}, 81.14^{\circ}, 64.9 \mathrm{~ns}\right), \\
l=4: & \left(81.87^{\circ}, 98.05^{\circ}, 98.13^{\circ}, 81.95^{\circ}, 71.4 \mathrm{~ns}\right) .
\end{array}
$$

The specular paths are deteriorated by undesired scattering. We draw 20 scattering points per specular reflection, as illustrated in Figure 4. The noise parameters are calculated based on the scattering point locations. In our evaluation we consider various levels of power ratios between power of specular and diffuse scattering paths.

It is worth noting that the multipath parameters are calculated based on a realistic environment setup where both transmitter and receiver are located at similar heights. Hence, the multipath parameters are not well separated. Strong similarities can be observed at the delay and elevation domain which makes the parameter estimation sensitive to additive noise. Figure 5 illustrates the RMS error of angles (left) and delays (right) for various levels of specular-to-scattering power ration (SSPR). At high SSPRs the paths are resolvable. Reducing the SSPR yields an increased RMS error. At SSPRs below 3 the algorithm is not able to separate paths $l=3$ and $l=4$ anymore.
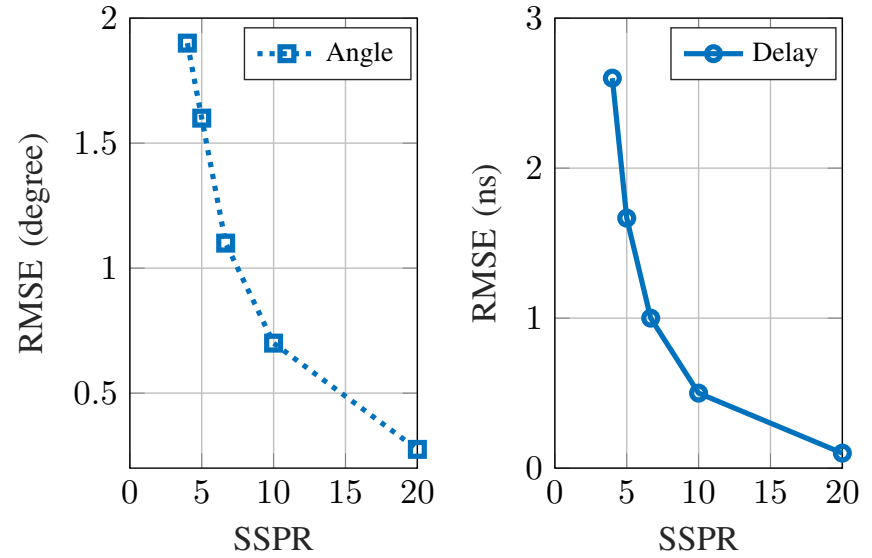

Fig. 5. RMSE error for angle (left) and delay estimation (right) under the Lambertian scattering model.

\section{CONCLUSIONS}

We propose a search-free $R$-D beamspace tensor-ESPRIT algorithm for mmWave MIMO channel estimation. It is a generalization of beamspace-ESPRIT method from matrix to tensor framework. The 5-D channel parameters are automatically associated. The performance of the proposed algorithm is evaluated by considering different precoders and combiners. Furthermore, the effect of the size of the precoder and combiner is also investigated by considering both the reflectors and scatters.

\section{ACKNOWLEDGMENTS}

This work was supported, in part, by European Commission H2020-MSCA-IF-2015 project No. 700044 (MassLOC) and H2020-ICT-2016-2 project No. 761510 (5GCAR) as well as the State Key Laboratory of Automotive Safety and Energy under Project No. KF1804. 


\section{APPENDIX A}

PROOF OF SHIFT INVARIANCE STRUCTURE IN BEAMSPACE

From (33), we have

$$
\mathbf{Q}_{r} \mathbf{W}_{r}^{H}=\mathbf{Q}_{r} \mathbf{W}_{r}^{H} \mathbf{J}_{r_{1}}^{H} \mathbf{J}_{r_{1}} .
$$

Multiplying $\mathbf{A}_{r}$ from left side of (44), we obtain

$$
\mathbf{Q}_{r} \mathbf{B}_{r}=\mathbf{Q}_{r} \mathbf{W}_{r}^{H} \mathbf{A}_{r}=\mathbf{Q}_{r} \mathbf{W}_{r}^{H} \mathbf{J}_{r_{1}}^{H} \mathbf{J}_{r_{1}} \mathbf{A}_{r} .
$$

Since

$$
\mathbf{W}_{r}^{H} \mathbf{J}_{r_{1}}^{H}=\mathbf{F}_{r}^{H} \mathbf{W}_{r}^{H} \mathbf{J}_{r_{2}}^{H}
$$

and

$$
\mathbf{J}_{r_{1}} \mathbf{A}_{r}=\mathbf{J}_{r_{2}} \mathbf{A}_{r} \boldsymbol{\Phi}_{r}
$$

Substituting (46) and (47) into (45), we have

$$
\mathbf{Q}_{r} \mathbf{B}_{r} \boldsymbol{\Phi}_{r}^{H}=\mathbf{Q}_{r} \mathbf{F}_{r}^{H} \mathbf{W}_{r}^{H} \mathbf{J}_{r_{2}}^{H} \mathbf{J}_{r_{2}} \mathbf{A}_{r}
$$

Similar to (44), we have

$$
\mathbf{Q}_{r} \mathbf{F}_{r}^{H} \mathbf{W}_{r}^{H}=\mathbf{Q}_{r}^{H} \mathbf{F}_{r}^{H} \mathbf{W}_{r}^{H} \mathbf{J}_{r_{2}}^{H} \mathbf{J}_{r_{2}} .
$$

Multiplying matrix $\mathbf{A}_{r}$ on both sides of (49), we have

$$
\mathbf{Q}_{r} \mathbf{F}_{r}^{H} \mathbf{B}_{r}=\mathbf{Q}_{r}^{H} \mathbf{F}_{r}^{H} \mathbf{W}_{r}^{H} \mathbf{J}_{r_{2}}^{H} \mathbf{J}_{r_{2}} \mathbf{A}_{r} .
$$

Comparing (48) and (50), we have

$$
\begin{gathered}
\mathbf{Q}_{r} \mathbf{F}_{r}^{H} \mathbf{B}_{r}=\mathbf{Q}_{r} \mathbf{B}_{r} \boldsymbol{\Phi}_{r}^{H} . \\
\text { REFERENCES }
\end{gathered}
$$

\section{REFERENCES}

[1] A. Shahmansoori, G. E. Garcia, G. Destino, G. Seco-Granados, and $\mathrm{H}$. Wymeersch, "Position and orientation estimation through millimeterwave MIMO in 5g systems," IEEE Transactions on Wireless Communications, vol. 17, no. 3, pp. 1822-1835, Mar. 2018.

[2] A. B. Gershman, M. Rübsamen, and M. Pesavento, "One-and twodimensional direction-of-arrival estimation: An overview of search-free techniques," Signal Processing, vol. 90, no. 5, pp. 1338-1349, 2010.

[3] R. Schmidt, "Multiple emitter location and signal parameter estimation," IEEE Transactions on Antennas and Propagation, vol. 34, no. 3, pp. 276-280, Mar. 1986.

[4] R. Roy and T. Kailath, "ESPRIT-estimation of signal parameters via rotational invariance techniques," IEEE Transactions on Acoustics, Speech, and Signal Processing, vol. 37, no. 7, pp. 984-995, Jul. 1989.

[5] Y. Hua and T. K. Sarkar, "Matrix pencil method for estimating parameters of exponentially damped/undamped sinusoids in noise," IEEE Transactions on Acoustics, Speech, and Signal Processing, vol. 38, no. 5 , pp. 814-824, May 1990.

[6] H. C. So, F. K. W. Chan, W. H. Lau, and C.-F. Chan, "An efficient approach for two-dimensional parameter estimation of a single-tone," IEEE Transactions on Signal Processing, vol. 58, no. 4, pp. 1999-2009, Apr. 2010.

[7] A. Cichocki, D. Mandic, L. D. Lathauwer, G. Zhou, Q. Zhao, C. Caiafa, and H. A. PHAN, "Tensor decompositions for signal processing applications: From two-way to multiway component analysis," IEEE Signal Processing Magazine, vol. 32, no. 2, pp. 145-163, Mar. 2015.

[8] L. Yang, J. Fang, H. Li, and B. Zeng, "An iterative reweighted method for tucker decomposition of incomplete tensors," IEEE Transactions on Signal Processing, vol. 64, no. 18, pp. 4817-4829, Sep. 2016.

[9] T. G. Kolda and B. W. Bader, "Tensor decompositions and applications," SIAM Review, vol. 51, no. 3, pp. 455-500, Aug. 2009.

[10] H. L. Van Trees, Optimum Array Processing. John Wiley \& Sons, Inc., Mar. 2002.

[11] J. Liu, X. Liu, and X. Ma, "Multidimensional frequency estimation with finite snapshots in the presence of identical frequencies," IEEE Transactions on Signal Processing, vol. 55, no. 11, pp. 5179-5194, Nov. 2007.
[12] M. Haardt, F. Roemer, and G. Del Galdo, "Higher-order SVD-based subspace estimation to improve the parameter estimation accuracy in multidimensional harmonic retrieval problems," IEEE Transactions on Signal Processing, vol. 56, no. 7, pp. 3198-3213, Jul. 2008.

[13] R. W. Heath, N. González-Prelcic, S. Rangan, W. Roh, and A. M. Sayeed, "An overview of signal processing techniques for millimeter wave MIMO systems," IEEE Journal of Selected Topics in Signal Processing, vol. 10, no. 3, pp. 436-453, Apr. 2016.

[14] Z. Tian and H. L. V. Trees, "Beamspace MODE," in Proc. Thirty-Fifth Asilomar Conference on Signals, Systems and Computers, vol. 2, Pacific Grove, California, Nov. 2001, pp. 926-930.

[15] M. D. Zoltowski, G. M. Kautz, and S. D. Silverstein, "Beamspace rootmusic," IEEE Transactions on Signal Processing, vol. 41, no. 1, pp. 344-364, Jan. 1993.

[16] J. Zhang and M. Haardt, "Channel estimation and training design for hybrid multi-carrier mmwave massive MIMO systems: The beamspace ESPRIT approach," in Proc. 25th European Signal Processing Conference, 2017, pp. 385-389.

[17] Z. Zhou, J. Fang, L. Yang, H. Li, Z. Chen, and R. S. Blum, "Lowrank tensor decomposition-aided channel estimation for millimeter wave MIMO-OFDM systems," IEEE Journal on Selected Areas in Communications, vol. 35, no. 7, pp. 1524-1538, Jul. 2017.

[18] G. Xu, S. D. Silverstein, R. H. Roy, and T. Kailath, "Beamspace ESPRIT," IEEE Transactions on Signal Processing, vol. 42, no. 2, pp. 349-356, Feb. 1994.

[19] J. A. Gansman, M. D. Zoltowski, and J. V. Krogmeier, "Multidimensional multirate DOA estimation in beamspace," IEEE Transactions on Signal Processing, vol. 44, no. 11, pp. 2780-2792, Nov. 1996.

[20] L. De Lathauwer, B. D. Moor, and J. Vandewalle, "A multilinear singular value decomposition," SIAM Journal on Matrix Analysis and Applications, vol. 21, no. 4, pp. 1253-1278, 2000.

[21] Y. Xu, "Fast algorithms for higher-order singular value decomposition from incomplete data," Journal of Computational Mathematics, vol. 35, no. 4, pp. 395-420, 2017.

[22] L. D. Lathauwer, B. D. Moor, and J. Vandewalle, "On the best rank1 and rank- $\left(R_{1}, R_{2}, \cdots, R_{N}\right)$ approximation of higher-order tensors," SIAM Journal on Matrix Analysis and Applications, vol. 21, no. 4, pp. 1324-1342, Jan. 2000.

[23] F. Roemer, M. Haardt, and G. D. Galdo, "Analytical performance assessment of multi-dimensional matrix- and tensor-based ESPRIT-type algorithms," IEEE Transactions on Signal Processing, vol. 62, no. 10, pp. 2611-2625, May 2014.

[24] T. Fu and X. Gao, "Simultaneous diagonalization with similarity transformation for non-defective matrices," in Proc. IEEE International Conference on Acoustics Speech and Signal Processing, Toulouse, France, May 2006, pp. 1137-1140.

[25] M. Haardt and J. A. Nossek, "Simultaneous Schur decomposition of several nonsymmetric matrices to achieve automatic pairing in multidimensional harmonic retrieval problems," IEEE Transactions on Signal Processing, vol. 46, no. 1, pp. 161-169, Jan. 1998.

[26] S. Valaee, B. Champagne, and P. Kabal, "Parametric localization of distributed sources," IEEE Transactions on Signal Processing, vol. 43, no. 9, pp. 2144-2153, Sep. 1995

[27] V. Degli-Esposti, F. Fuschini, E. M. Vitucci, and G. Falciasecca, "Measurement and modelling of scattering from buildings," IEEE Transactions on Antennas and Propagation, vol. 55, no. 1, pp. 143-153, 2007. 